\title{
Article \\ Association of Lumbar Paraspinal Muscle Morphometry with Degenerative Spondylolisthesis
}

\author{
Eun Taek Lee ${ }^{1}$, Seung Ah Lee ${ }^{1}$, Yunsoo Soh ${ }^{1}{ }^{1}$, Myung Chul Yoo ${ }^{1}$, Jun Ho Lee ${ }^{2}{ }^{\circledR}$ and Jinmann Chon ${ }^{1, *}$ \\ 1 Department of Physical Medicine \& Rehabilitation, College of Medicine, Kyung Hee University, \\ Seoul 02447, Korea; mylot91@naver.com (E.T.L.); Isarang80@gmail.com (S.A.L.); soyuns@gmail.com (Y.S.); \\ famousir@naver.com (M.C.Y.) \\ 2 Department of Neurosurgery, College of Medicine, Kyung Hee University, Seoul 02447, Korea; \\ moo9924@khu.ac.kr \\ * Correspondence: kkangmann@naver.com; Tel.: +82-2-958-8565; Fax: +82-2-958-8560
}

Citation: Lee, E.T.; Lee, S.A.; Soh, Y.; Yoo, M.C.; Lee, J.H.; Chon, J. Association of Lumbar Paraspinal Muscle Morphometry with Degenerative Spondylolisthesis. Int. J. Environ. Res. Public Health 2021, 18, 4037. https://doi.org/10.3390/ ijerph18084037

Academic Editor: Joseph Marino

Received: 5 January 2021

Accepted: 30 March 2021

Published: 12 April 2021

Publisher's Note: MDPI stays neutra with regard to jurisdictional claims in published maps and institutional affiliations.

Copyright: (c) 2021 by the authors. Licensee MDPI, Basel, Switzerland. This article is an open access article distributed under the terms and conditions of the Creative Commons Attribution (CC BY) license (https:// creativecommons.org/licenses/by/ $4.0 /)$.

\begin{abstract}
The objective of this study was to assess the cross-sectional areas (CSA) of lumbar paraspinal muscles and their fatty degeneration in adults with degenerative lumbar spondylolisthesis (DLS) diagnosed with chronic radiculopathy, compare them with those of the same age- and sex-related groups with radiculopathy, and evaluate their correlations and the changes observed on magnetic resonance imaging (MRI). This retrospective study included 62 female patients aged 65-85 years, who were diagnosed with lumbar polyradiculopathy. The patients were divided into two groups: 30 patients with spondylolisthesis and 32 patients without spondylolisthesis. We calculated the CSA and fatty degeneration of the erector spinae (ES) and multifidus (MF) on axial T2-weighted magnetic resonance (MR) images from the inferior end plate of the L4 vertebral body levels. The functional CSA (FCSA): CSA ratio, skeletal muscle index (SMI), and MF CSA: ES CSA ratio were calculated and compared between the two groups using an independent $t$-test. We performed logistic regression analysis using spondylolisthesis as the dependent variable and SMI, FCSA, rFCSA, fat infiltration rate as independent variables. The result showed more fat infiltration of MF in patients with DLS ( 56.33 vs. $44.66 \% ; p=0.001$ ). The mean FCSA (783.33 vs. $666.22 \mathrm{~mm}^{2} ; p=0.028$ ) of ES muscle was a statistically larger in the patients with DLS. The ES FCSA / total CSA was an independent predictor of lumbar spondylolisthesis (odd ratio $=1.092, p=0.016$ ), while the MF FCSA / total CSA was an independent protective factor (odd ratio $=0.898, p=0.002$ )
\end{abstract}

Keywords: degenerative spondylolisthesis; polyradiculopathy; erector spinae; multifidus; paraspinal muscles; fatty degeneration; cross sectional area; lumbar

\section{Introduction}

Degenerative lumbar spondylolisthesis (DLS) is a disorder that causes the slip of one vertebral body (VB) over the other one below due to degenerative changes in the spine. The reported incidence of DS varies among studies, from $4.1 \%$ in cadaveric material, $13.6 \%$ in a population-based cohort study, and up to $28.6 \%$ in a clinical cohort study [1,2]. DLS is the major cause of spinal canal stenosis and is often associated with back and leg pain [3].

There are some known characteristics of DLS. The male-to-female ratio of DLS was $1: 5-6$. DLS is uncommon in patients aged $<50$ years. The most commonly affected location is the L4/L5 level, and the slip rarely exceeds 30\% of the width of the subjacent vertebra [4]. The possible causative factors of DLS are the degree of pelvic inclination and declination, iliolumbar ligament strength, degree of adjacent disk or facet joint degeneration, and relative L1-S1 angle [5]. Patients with DLS are usually asymptomatic, but the condition may progress to spinal stenosis [4].

Degenerative spondylolisthesis is associated with increased age in both sexes [4]. Aging leads to skeletal muscle mass atrophy, which can be replaced by connective tissues 
such as fat. These changes, known as sarcopenia, are due to a reduction in both the number and size of muscle fibers.

The lumbar muscles functionally support and maintain spine stability [6]. The multifidus muscle (MF) is the most medial part of the lumbar paraspinal muscles, innervated by the dorsal ramus medial branch of the segmental nerve [7]. The erector spinae muscle (ES) acts as a global muscle affecting movement and muscular strength [6]. During the last decade, an increasing number of studies have further explored the interaction among the paraspinal muscles, low back pain (LBP), and spinal pathology. Degeneration of the paraspinal muscle with decreased isometric force of the L-spine, low back pain, disk herniation, polyradiculopathy, and DLS have been reported in many studies measuring the cross-sectional area (CSA) of the paraspinal muscles [6,8-11]. Spinal stability is an important factor to consider in the evaluation and treatment of patients with DLS [12]. Therefore, evaluation of CSA of paraspinal muscle can be crucial because these muscles can directly influence segmental stability and control of the lumbar spine owing to their attachment to the spinal column.

In this study, the patient population comprised only women, aged 65-85 years, as age-related changes in skeletal muscle quality differ between the sexes [4]. Men have less fatty degeneration of paraspinal muscles compared to women; therefore investigation of fat infiltration in women may be needed first for a more accurate comparison. We hypothesized that the deficit in muscular stabilization caused by degeneration of the MF and ES is associated with DLS. However, when patients with DLS have chronic radiculopathy or disk degeneration, it is difficult to differentiate the correlation between MF atrophy. Electromyography assess denervation in the multifidus muscle more accurately and perhaps contribute to the acquisition of additional evidence to support the correlation between multifidus muscle fatty infiltration and structural parameters. Therefore, the current study assessed the CSA of the lumbar paraspinal muscles and degree of fat infiltration into the MF and ES in adults with DLS and chronic radiculopathy. We aimed to determine the association between DLS and CSA of the paraspinal muscle by precluding the factors that can influence the CSA of the paraspinal muscle.

\section{Methods}

\subsection{Subjects}

We, retrospectively, analyzed 83 women aged 65-85 years, who were diagnosed with lumbar polyradiculopathy on electrodiagnostic study from January 2017 to April 2020 at the clinic of the Department of Rehabilitation Medicine of Kyung Hee University Hospital. The inclusion criteria were: (1) female sex, (2) an age of 65-85 years, (3) presence of L-spine MRI study, and (4) polyradiculopathy at the L-spine level. The electrodiagnostic criteria for radiculopathy were abnormal spontaneous activity, abnormal motor unit morphology consistent with nerve injury (polyphasic, large amplitude, increased duration), and reduced recruitment patterns in muscles innervated by the same myotome but different muscles. Patients with polyneuropathy, previous lumbosacral spinal surgery, systemic disorders, spinal fractures, tumors, or infections were excluded. Approval for this study was obtained from the institutional review board (IRB; IRB number: 2020-12-048).

In all, 83 patients were eligible based on their electrodiagnostic findings. All patients had radiculopathy between the L3 and S1 levels, and 17 patients had unilateral polyradiculopathy (22.6\%). A total of nine patients had an ongoing denervation pattern; another 21 patients were excluded from the study. Finally, 62 patients were selected and divided into two groups based on their radiologic findings: patients with spondylolisthesis (30 cases) and those without spondylolisthesis (32 cases) (Figure 1). 


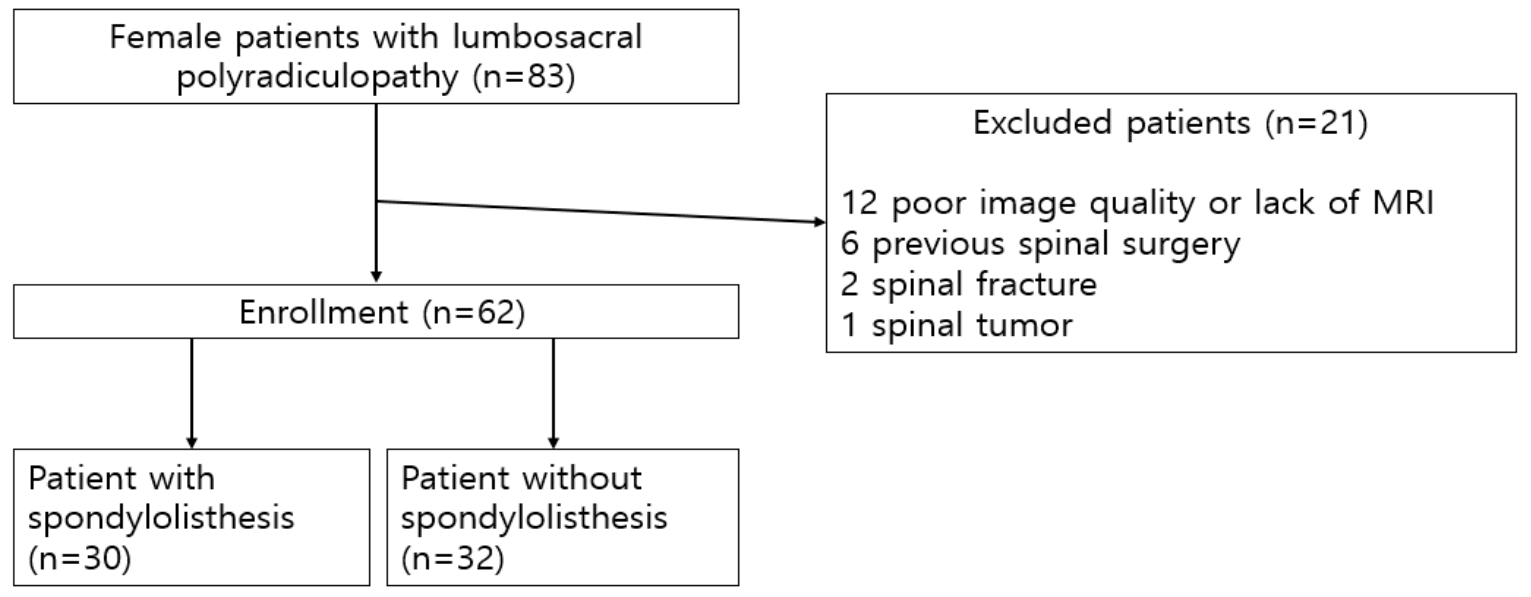

Figure 1. Flow chart of patient enrollment and grouping.

\subsection{Measurements and Procedures}

All patients underwent lumbosacral spine magnetic resonance imaging (MRI). MRI and electromyography study days were not separated by more than 6 weeks. The degree of spondylolisthesis was determined according to the Meyerding classification. Disk degeneration was graded on a 5-point ordinal scale, as described by Pfirrmann et al. [13]. Facet arthropathy at the level of the listhesis was recorded as a categorical variable based on the established MRI criteria [14]. Axial T2-weighted MRI images were obtained at the level of the L4 inferior endplate. At the L4/L5 disk level, the anterior and posterior middle disk heights were measured to evaluate the height disk [15]. The CSAs of both sides of the MF and ES and L4 inferior endplate margin were measured by drawing their outlines with the region of interest (ROI) using a PiView program (Infinitt, Seoul, Korea). The facet arthropathy grade was measured.

Axial T2-weighted MR images were exported from a picture archiving and communication system (PACS) workstation and reformatted using image processing software (Image J, version 1.53e, National Institutes of Health, Wayne Rasband, Washington, DC, USA). Quantitative measurements of the erector spinae and multifidus muscles were obtained for each patient using a method previously proposed by Fortin et al. [16].

CSA was defined by manually tracing the fascial boundary of the multifidus and erector spinae bilaterally. Functional CSA (FCSA) measurements were obtained using a highly reliable thresholding technique, which is based on the difference in signal intensity between muscle (low signal) and fat tissue (high signal), allowing for the separation of both tissues. Total CSA and FCSA were measured individually for the MF and ES muscles. The reliability of the FCSA and total CSA measurements was relatively equivalent across all spinal levels [17]. FCSA was measured by selecting a threshold signal intensity within the total muscle CSA to include only pixels within the lean muscle range (Figure 2). The grayscale range for lean muscle tissue was selected for every patient in each slice. The six sample ROIs within the bilateral paraspinal muscle were taken from areas of lean muscle tissue visible on each slice, avoiding the inclusion of any visible pixel of fat (Figure 2). The maximum signal intensity acquired from the six ROIs was selected as the highest threshold to distinguish muscle tissue from fat. Subsequently, the percentage of red area (ratio of FCSA to total CSA) in the muscle compartment was calculated (Figure 2). The fat infiltration rate can be calculated (100\%- ratio of FCSA to total CSA\%). 


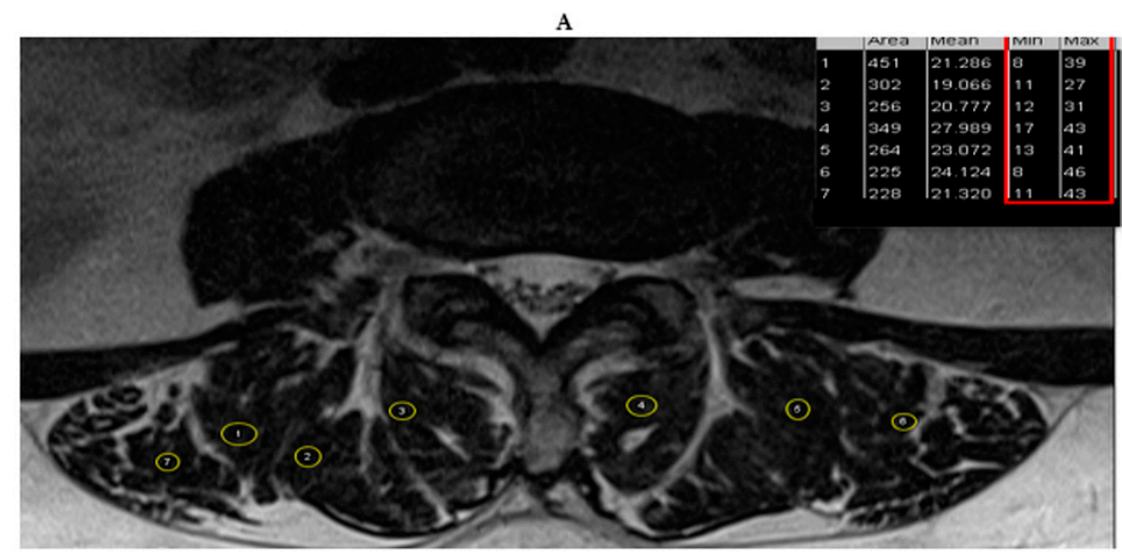

B

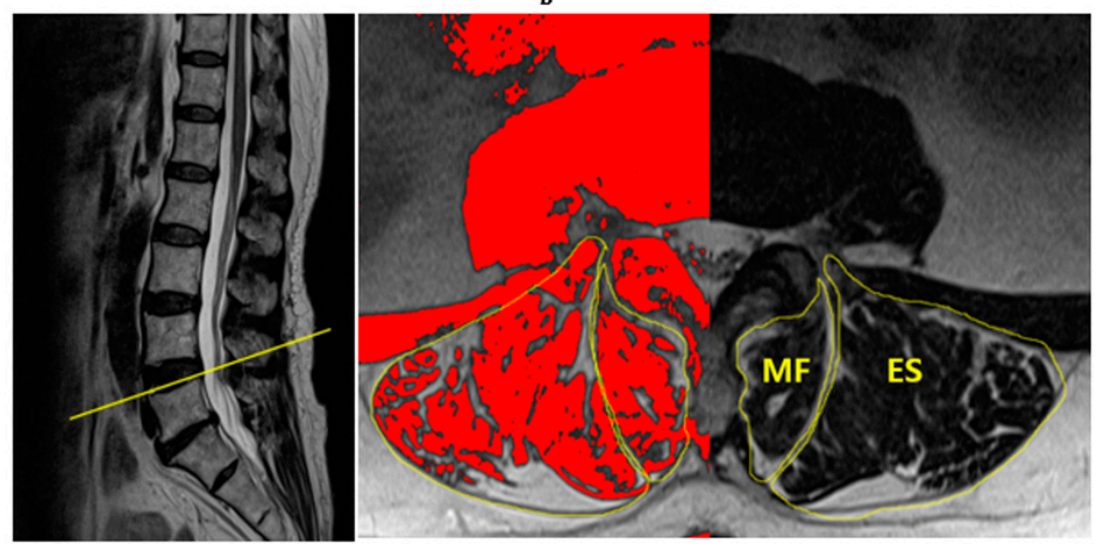

Figure 2. Quantitative paraspinal muscle measurement. Measurement of cross-sectional area (CSA) and functional CSA of the multifidus muscle (MF) and erector spinae (ES) muscle at the L4-L5 disk level. (A) Sample selection of regions of interest (ROIs) to define upper and lower signal intensity threshold limits. (B) Sagittal and axial T2 weighted MR image of the FCSA of the paraspinal muscle group (red area), using the threshold method with ROI tracing.

The previous two studies showed multifidus atrophy and erector spinae hypertrophy in patients with DLS, we first calculated the MF: ES ratio as a predictive factor for DLS $[18,19]$. Previous studies have used the relative CSA (rCSA) to compensate for the influence of individual body shape, body weight, and height on the CSA of the muscles [6]. Therefore, we calculated the rCSA of the muscles, which is defined as the ratio of the CSA of muscles to that of the lower margin of the L4 vertebra. In another previous study on paraspinal lean muscle measurement, the skeletal muscle index (SMI) was used to normalize values across varying patient heights, akin to the body mass index (BMI) calculation [20]. For example, SMI of total CSA = total CSA of muscle on an axial scan (expressed as $\mathrm{cm}^{2}$ ) divided by the square of the patient's height (expressed as $\mathrm{cm}^{2}$ ).

All CSA measurements were performed independently twice, with a 1-week interval, by the first author (E.T.L.) to minimize the potential for error in constructing the polygons around the margins of muscles. The average values of the two measurements were used for statistical analysis. The clinical and electrodiagnostic findings and DLS group of all cases were blinded. The measurement of CSA, disk degeneration grade, facet arthropathy grade, disk height were performed by the first author (E.T.L.) with 4 years of experience in spinal MRI measurement.

\subsection{Statistical Analysis}

Statistical analysis was performed using the SPSS ver. 25.0 for Windows (IBM Corp., Armonk, NY, USA). Cohen's effect size and power was calculated using G Power ver.3.1. We performed an independent $t$-test and Fisher's exact test to analyze the differences in 
the demographic and radiologic differences. An independent t-test was used to investigate statistical relationships of total CSA, rCSA, FCSA, FCSA/total CSA (\%), MF: ES ratio, and SMI between the two groups. These variables were entered into binary logistic regression analysis. To determine which variables were the most statistically appropriate independent predictors (largest area under the curve (AUC) area) of DLS, we performed five logistic regression analyses. We used DLS as the dependent variable and five sets of variables (MF FCSA/ES FCSA, SMI FCSA, ratio of FCSA to total CSA, FCSA, rFCSA) as the independent variables. BMI, age, disk height, facet arthropathy grade, disk degeneration grade were adjusted in each logistic regression analysis. Statistical significance was set at $p$-values less than 0.05 . All values were presented as mean \pm standard deviation.

\section{Results}

\subsection{Characteristics of Participants}

There was no statistically significant differences in age, height, weight, BMI, or L4/L5 disk height between the two groups (Table 1).

Table 1. Demographic and radiological characteristics of patients.

\begin{tabular}{cccc}
\hline Sex & $\begin{array}{c}\text { Patients with } \\
\text { Spondylolisthesis } \\
(\mathbf{n}=\mathbf{3 0})\end{array}$ & $\begin{array}{c}\text { Patients without } \\
\text { Spondylolisthesis } \\
(\mathbf{n}=\mathbf{3 2})\end{array}$ & $p$ \\
\hline Age (years) & $74.1 \pm 5.6$ & $74.5 \pm 5.4$ & 0.727 \\
Height (cm) & $150.6 \pm 6.4$ & $151.7 \pm 6.3$ & 0.511 \\
Weight (kg) & $56.8 \pm 7.1$ & $57.3 \pm 11.0$ & 0.861 \\
BMI (kg/m $\left.{ }^{2}\right)$ & $25.0 \pm 2.6$ & $24.8 \pm 4.1$ & 0.801 \\
L4-L5 disc height & $9.4 \pm 2.7$ & $9.47 \pm 2.8$ & 0.949 \\
$(\mathrm{~mm})$ & & & \\
\hline
\end{tabular}

\subsection{MRI Findings}

In the spondylolisthesis group, 19 patients (86.3\%) had L4/L5 or L5/S1 spondylolisthesis, and 29 patients $(96.6 \%)$ had grade I spondylolisthesis (Table 2). Disk degeneration and L4/L5 facet arthropathy grades were measured, there were no statistically significant differences in disk degeneration and facet arthropathy grade between two groups (Table 3).

Table 2. Radiologic features spondylolisthesis group.

\begin{tabular}{cc}
\hline Radiologic Features & Number of Patients (\%) \\
\hline Level of listhesis & \\
L3-4 & $2(6.7 \%)$ \\
L4-5 & $19(63.34 \%)$ \\
L5-S1 & $9(30.0 \%)$ \\
Listhesis grade & \\
grade 1 & $29(96.6 \%)$ \\
grade 2 & $1(3.3 \%)$ \\
grade 3 & 0 \\
grade 4 & 0 \\
\hline
\end{tabular}

For the MF muscle, the mean total CSA, rCSA, rFCSA, and SMI of total CSA showed no statistically significant difference between the two groups. However, the mean FCSA of the MF was significantly smaller in patients with DLS (244.63 vs. $298.15 \mathrm{~mm}^{2} ; p=0.030$ ). The mean FCSA: total CSA ratio ( $43.67 \mathrm{vs.} 55.34 \% ; p=0.001$ ) showed a statistically significant difference between two groups (Cohen's Effect size d: 0.876, power: 0.924$)$. This means, the result showed more fat infiltration of MF in patients with DLS (56.33 vs. $44.66 \% ; p=0.001$ ). The mean SMI of the FCSA (107.44 vs. $129.79 \mathrm{~mm}^{2} / \mathrm{m}^{2} ; p=0.38$ ) were significantly lower in patients with DLS than in patients without DLS. 
Table 3. Radiologic features of 62 patients.

\begin{tabular}{llll}
\hline & $\begin{array}{l}\text { Patients with } \\
\text { Spondylolisthesis } \\
(\mathbf{n}=\mathbf{3 0})\end{array}$ & $\begin{array}{l}\text { Patients without } \\
\text { Spondylolisthesis } \\
(\mathbf{n = 3 2 )}\end{array}$ & $p$ \\
\hline Disc degeneration grade & & & \\
1 & 0 & 0 & \\
2 & 0 & 0 & \\
3 & 2 & 1 & \\
4 & 14 & 16 & \\
5 & 14 & 15 & \\
L4/L5 facet arthropathy grade & & & \\
0 & 0 & 0 & \\
1 & 0 & 3 & \\
2 & 11 & 16 & \\
3 & 19 & 13 & \\
\hline
\end{tabular}

For the ES muscle, there were no statistically significant differences between two groups in the mean total CSA, rCSA, rFCSA, ratio of FCSA: total CSA, and SMI of total CSA. However, the mean FCSA (783.33 vs. $666.22 \mathrm{~mm}^{2} ; p=0.028$ ) and SMI FCSA (343.95 vs. $288.78 \mathrm{~mm}^{2} / \mathrm{m}^{2} ; p=0.012$ ) of ES muscle was a statistically larger in the patients with DLS.

There were no statistically significant differences in MF total, CSA: ES total, and CSA ratio between the two groups. However, the mean MF FCSA: ES FCSA ratio showed a statistically significant difference between the two groups ( 0.33 vs. $0.45 ; p<0.001)$ (Table 4). Patients with DLS showed much smaller MF FCSA to ES FCSA ratio.

Table 4. Magnetic resonance imaging findings for multifidus and erector spinae muscle groups by degenerative lumbar spondylolisthesis.

\begin{tabular}{|c|c|c|c|}
\hline Muscle Group & $\begin{array}{l}\text { Patients with } \\
\text { Spondylolisthesis } \\
(\mathrm{n}=30)\end{array}$ & $\begin{array}{l}\text { Patients without } \\
\text { Spondylolisthesis } \\
(\mathrm{n}=32)\end{array}$ & $p$ \\
\hline \multicolumn{4}{|l|}{ Multifidus muscle group } \\
\hline Total CSA $\left(\mathrm{mm}^{2}\right)$ & $553.80 \pm 136.59$ & $556.25 \pm 157.27$ & 0.948 \\
\hline $\operatorname{FCSA}\left(\mathrm{mm}^{2}\right)$ & $244.63 \pm 101.39$ & $298.15 \pm 88.48$ & 0.030 \\
\hline Ratio of FCSA to total CSA (\%) & $43.67 \pm 13.33$ & $55.34 \pm 13.31$ & 0.001 \\
\hline CSA/VB (rCSA) & $0.473 \pm 0.395$ & $0.365 \pm 0.156$ & 0.162 \\
\hline FCSA/VB (rFCSA) & $0.202 \pm 0.203$ & $0.199 \pm 0.097$ & 0.940 \\
\hline \multicolumn{4}{|l|}{$\operatorname{SMI}\left(\mathrm{mm}^{2} / \mathrm{m}^{2}\right)$} \\
\hline of Total CSA & $244.09 \pm 60.56$ & $242.26 \pm 68.94$ & 0.912 \\
\hline of FCSA & $107.44 \pm 43.91$ & $129.79 \pm 39.11$ & 0.038 \\
\hline \multicolumn{4}{|l|}{ Erector spinae muscle group } \\
\hline Total CSA $\left(\mathrm{mm}^{2}\right)$ & $1511.05 \pm 273.26$ & $1384.7 \pm 281.06$ & 0.078 \\
\hline $\mathrm{FCSA}\left(\mathrm{mm}^{2}\right)$ & $783.33 \pm 225.09$ & $666.22 \pm 182.28$ & 0.028 \\
\hline Ratio of FCSA to total CSA (\%) & $51.69 \pm 11.79$ & $48.57 \pm 10.94$ & 0.283 \\
\hline CSA/VB (rCSA) & $1.225 \pm 0.975$ & $0.879 \pm 0.261$ & 0.058 \\
\hline FCSA/VB (rFCSA) & $0.643 \pm 0.586$ & $0.432 \pm 0.180$ & 0.057 \\
\hline \multicolumn{4}{|l|}{$\operatorname{SMI}\left(\mathrm{mm}^{2} / \mathrm{m}^{2}\right)$} \\
\hline of Total CSA & $667.11 \pm 124.70$ & $604.71 \pm 128.65$ & 0.057 \\
\hline of FCSA & $343.95 \pm 94.06$ & $288.78 \pm 72.23$ & 0.012 \\
\hline MF CSA/ES CSA & $0.37 \pm 0.98$ & $0.45 \pm 0.23$ & 0.062 \\
\hline MF FCSA/ES FCSA & $0.33 \pm 0.15$ & $0.45 \pm 0.12$ & 0.000 \\
\hline
\end{tabular}

CSA, cross-sectional area; FCSA, functional CSA; SMI, skeletal muscle index; VB, vertebral body; MF, multifidus; ES, erector spinae.

After applying logistic regression analysis, all variables, except rFCSA $(p=0.822)$, correlated significantly with patients with DLS. Among five sets of variables, MF FCSA/total CSA with ES FCSA/total CSA showed largest AUC area (0.827). 
The ES FCSA / total CSA was an independent predictor of lumbar spondylolisthesis $(\mathrm{OR}=1.092, p=0.016)$, while the MF FCSA/total CSA was an independent protective factor $(\mathrm{OR}=0.898, p=0.002)($ Table 5$)$.

Table 5. Logistic regression analysis: factors associated with the occurrence of degenerative lumbar spondylolisthesis

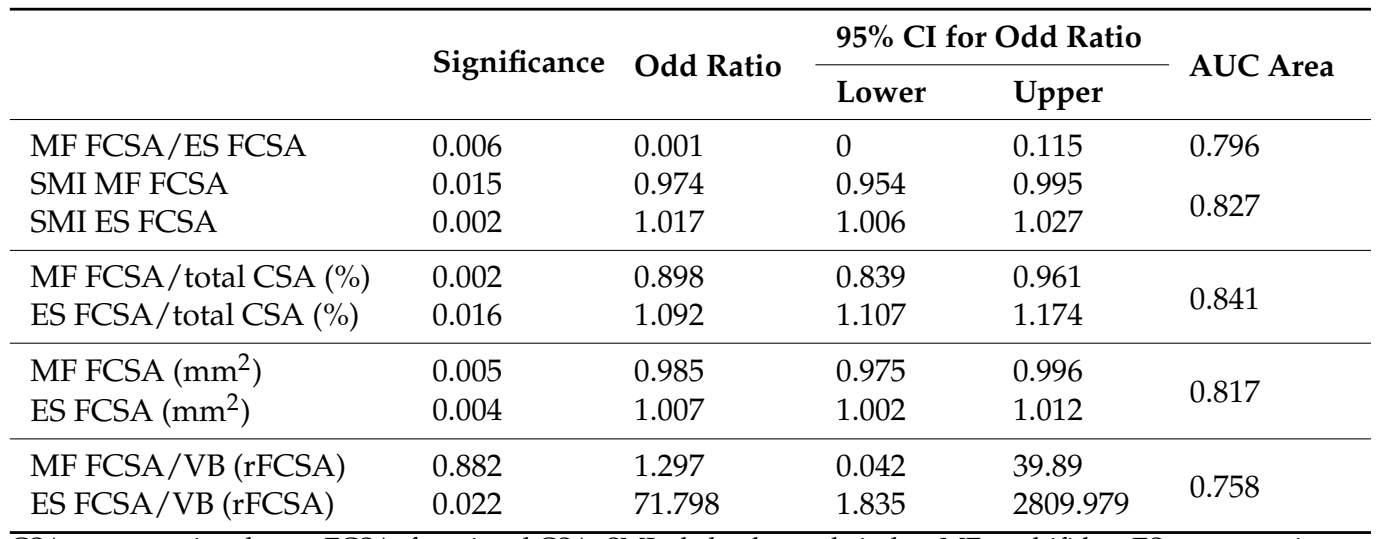

CSA, cross-sectional area; FCSA, functional CSA; SMI, skeletal muscle index; MF, multifidus; ES, erector spinae.

\section{Discussion}

In this study, patients with radiculopathy showed no significant difference in total CSA of the MF or ES. Instead, the fat infiltration ratio (100-FCSA / total CSA\%) and functional CSA of the MF were significantly different between those with and without DLS. The patients with DLS showed more fat infiltration in multifidus muscle and small FCSA. In contrast, erector spinae muscle showed a large FCSA in patients with DLS. This result is consistent with that of a previous study on fat infiltration. Fat infiltration is a sign of muscle atrophy, and the replacement of muscle with fat, while changing the function of the muscle, may not significantly alter its CSA [21].

Several studies have investigated the association between spondylolisthesis and paraspinal muscle CSA [22]. In a computer tomography (CT) study, a significant association was found between the density of the multifidus muscle at level L4 and spondylolisthesis [23]. A previous Chinese study evaluated the MRIs of 149 middle-aged patients with degenerative spondylolisthesis and age- and sex-matched controls. The MF muscle atrophy ratio (lean CSA: total CSA) of the study group tended to be significantly lower than that of the control group, whereas the signal intensity of the paraspinal muscles and the ES muscle atrophy ratio were significantly higher in the former than in the latter [19].

Thakar et al. assessed the CSA of lumbar paraspinal muscles in 120 adults with isthmic spondylolisthesis. The mean CSA value of the ES was significantly higher in the study cohort $(p=0.002)$ than in age- and sex-matched controls, whereas the CSA of the MF muscle was significantly lower $(p=0.009)$ [19].

In this study, patients with DLS showed high mean ES FCSA and SMI FCSA. This means that larger lean muscle volume of erector spinae was found in patients with DLS. We can cautiously conclude that patients with spondylolisthesis suffer from segmental atrophy of the MF muscle. ES hypertrophy may compensate for this instability [22].

The MF muscle is divided into five myotomes, each innervated by a single spinal segment. Muscle fibers attached to the spinous process of a particular vertebra are segmentally innervated by the medial branch of the dorsal ramus, which originates inferior to the respective vertebra [19]. The deep fiber of the MF is composed of type I (slow-twitch) fibers. It is suitable for low-load tonic activity. It is more vulnerable to immobilization or pain than type II (fast-twitch) fibers and can be considered as one of the reasons for selective atrophy of MF [19].

This study compared various measures. The fat infiltration ratio and rFCSA was investigated in patients with DLS in previous studies; SMI, MF:ES ratio were first applied 
in this study. However, to our knowledge, this is the first study to compare these variables simultaneously. Furthermore, we attempted to find the best variable to predict patient with DLS. In this study, increased fat infiltration in the multifidus muscle with decreased fat infiltration in the erector spinae muscle are the best predictors of the patient with DLS.

A strength of our study was the diagnosis of lumbosacral radiculopathy. Most of the previous studies used age- and sex-related normative populations as control groups, failing to exclude the influence of chronic radiculopathy-induced MF atrophy. Only our study included electrodiagnostic testing and patients with radiculopathy who were older than 65 years participated in this study. Furthermore, we used quantitative methods to accurately measure fat infiltration, as opposed to a previous study.

Our study provides a basis for spinal stabilization exercise and physiotherapy. The MF muscle is an important stabilizer of the lumbar spine neutral zone, and atrophy of the muscle decreases the ability to control the neutral zone and is strongly associated with LBP [24]. Spinal stabilization has been observed to be more effective over time in treating LBP than minimal intervention and exercise therapy alone. Therefore, the treatment focus has shifted to reactivation and strengthening of the smaller muscles of the spine to improve long-term stabilization of the vertebral column [24]. The benefit of targeted MF muscle strengthening programs utilizing this functional peculiarity has been demonstrated in patients with acute and chronic LBP with DLS. A study of subjects with acute back pain demonstrated normalization of the MF areas within a month after a specific motor re-education exercise program [11].

This study had several limitations to this study. First, the sample size was small, and only women were included in this study. Second, this was cross-sectional study and performed retrospectively. For future prospective studies, longitudinal data are needed to determine whether DLS is a cause or result of replacement of MF muscles with fat. Third, a standardized assessment of pain severity and daily activity was not performed. Medical treatment that leads to pain relief may also affect the structure of the lumbar muscles; this should be examined in future prospective studies.

\section{Conclusions}

Small FCSA of the multifidus muscle with high degree of fat infiltration and large FCSA of the erector spinae muscle were found in the DLS group with chronic radiculopathy.

MF FCSA/ES FCSA, SMI FCSA, ratio of FCSA to total CSA, and FCSA were independent predictors of lumbar spondylolisthesis, while increased fat infiltration in the multifidus muscle with decreased fat infiltration in erector spinae muscle best predict the patient with DLS.

Author Contributions: Conceptualization and data curation, E.T.L., J.C., and, J.H.L.; investigation, E.T.L. and J.C.; interpretation of data, E.T.L.; methodology, supervision, S.A.L., Y.S., M.C.Y., and J.C.; writing (original draft) E.T.L. All authors have read and agreed to the published version of the manuscript.

Funding: This research received no external funding.

Institutional Review Board Statement: Approval for this study was obtained from the Institutional Review Board (IRB) (IRB number: 2020-12-048).

Informed Consent Statement: Patient consent was waived due to the risk to study subjects is extremely low.

Data Availability Statement: The data presented in this study are available on request from the corresponding author. The data are not publicly available due to privacy matters.

Acknowledgments: We would like to express our gratitude to the medical staff of Kyung Hee University Medical Center for their helpful comments and technical assistance.

Conflicts of Interest: No potential conflicts of interest relevant to this article was reported. 


\section{References}

1. Farfan, H. The pathological anatomy of degenerative spondylolisthesis. A cadaver study. Spine 1980, 5, 412-418. [CrossRef]

2. Morgan, F.P.; King, T. Primary instability of lumbar vertebrae as a common cause of low back pain. J. Bone Joint Surgery 1957, 39, 6-22. [CrossRef]

3. Kalichman, L.; Hunter, D.J. Diagnosis and conservative management of degenerative lumbar spondylolisthesis. Eur Spine J 2008, 17, 327-335. [CrossRef] [PubMed]

4. Jacobsen, S.; Sonne-Holm, S.; Rovsing, H.; Monrad, H.; Gebuhr, P. Degenerative lumbar spondylolisthesis: An epidemiological perspective: The Copenhagen Osteoarthritis Study. Spine 2007, 32, 120-125. [CrossRef] [PubMed]

5. Fitzgerald, J.; Newman, P. Degenerative spondylolisthesis. J. Bone Joint Surgery 1976, 58, 184-192. [CrossRef] [PubMed]

6. Lee, H.J.; Lim, W.H.; Park, J.-W.; Kwon, B.S.; Ryu, K.H.; Lee, J.H.; Park, Y.G. The relationship between cross sectional area and strength of back muscles in patients with chronic low back pain. Ann. Rehabil. Med. 2012, 36, 173. [CrossRef]

7. Kader, D.; Wardlaw, D.; Smith, F. Correlation between the MRI changes in the lumbar multifidus muscles and leg pain. Clin. Radiol. 2000, 55, 145-149. [CrossRef] [PubMed]

8. Cooper, R.G.; Forbes, W.S.; Jayson, M.I.V. Radiographic Demonstration of Paraspinal Muscle Wasting in Patients with Chronic Low-Back-Pain. Brit. J. Rheumatol. 1992, 31, 389-394. [CrossRef] [PubMed]

9. Danneels, L.A.; Vanderstraeten, G.G.; Cambier, D.C.; Witrouw, E.E.; De Cuyper, H.J. CT imaging of trunk muscles in chronic low back pain patients and healthy control subjects. Eur. Spine J. 2000, 9, 266-272. [CrossRef]

10. Hides, J.; Stokes, M.; Saide, M.; Jull, G.; Cooper, D. Evidence of lumbar multifidus muscle wasting ipsilateral to symptoms in patients with acute/subacute low back pain. Spine 1994, 19, 165-172. [CrossRef]

11. Hides, J.A.; Richardson, C.A.; Jull, G.A. Multifidus muscle recovery is not automatic after resolution of acute, first-episode low back pain. Spine 1996, 21, 2763-2769. [CrossRef]

12. Simmonds, A.M.; Rampersaud, Y.R.; Dvorak, M.F.; Dea, N.; Melnyk, A.D.; Fisher, C.G. Defining the inherent stability of degenerative spondylolisthesis: A systematic review. J. Neurosurg. Spine 2015, 23, 178-189. [CrossRef] [PubMed]

13. Pfirrmann, C.W.A.; Metzdorf, A.; Zanetti, M.; Hodler, J.; Boos, N. Magnetic resonance classification of lumbar intervertebral disc degeneration. Spine 2001, 26, 1873-1878. [CrossRef] [PubMed]

14. Fujiwara, A.; Tamai, K.; Yamato, M.; An, H.S.; Yoshida, H.; Saotome, K.; Kurihashi, A. The relationship between facet joint osteoarthritis and disc degeneration of the lumbar spine: An MRI study. Eur. Spine J. 1999, 8, 396-401. [CrossRef] [PubMed]

15. Inoue, H.; Ohmori, K.; Miyasaka, K.; Hosoe, H. Radiographic evaluation of the lumbosacral disc height. Skelet. Radiol. 1999, 28, 638-643. [CrossRef] [PubMed]

16. Fortin, M.; Battié, M.C. Quantitative paraspinal muscle measurements: Inter-software reliability and agreement using OsiriX and ImageJ. Phys. Therapy 2012, 92, 853-864. [CrossRef]

17. Ranson, C.A.; Burnett, A.F.; Kerslake, R.; Batt, M.E.; O'Sullivan, P.B. An investigation into the use of MR imaging to determine the functional cross sectional area of lumbar paraspinal muscles. Eur. Spine J. 2006, 15, 764-773. [CrossRef] [PubMed]

18. Wang, G.; Karki, S.B.; Xu, S.; Hu, Z.; Chen, J.; Zhou, Z.; Fan, S. Quantitative MRI and X-ray analysis of disc degeneration and paraspinal muscle changes in degenerative spondylolisthesis. J. Back Musculoskelet 2015, 28, 277-285. [CrossRef]

19. Thakar, S.; Sivaraju, L.; Aryan, S.; Mohan, D.; Kiran, N.A.S.; Hegde, A.S. Lumbar paraspinal muscle morphometry and its correlations with demographic and radiological factors in adult isthmic spondylolisthesis: A retrospective review of 120 surgically managed cases. J. Neurosurg. Spine 2016, 24, 679-685. [CrossRef]

20. Chang, M.Y.; Park, Y.; Ha, J.W.; Zhang, H.Y.; Lee, S.H.; Hong, T.H.; Lee, S.H. Paraspinal Lean Muscle Mass Measurement Using Spine MRI as a Predictor of Adjacent Segment Disease After Lumbar Fusion: A Propensity Score-Matched Case-Control Analysis. AJR Am. J. Roentgenol. 2019, 1-8. [CrossRef]

21. Teichtahl, A.J.; Urquhart, D.M.; Wang, Y.; Wluka, A.E.; Wijethilake, P.; O'Sullivan, R.; Cicuttini, F.M. Fat infiltration of paraspinal muscles is associated with low back pain, disability, and structural abnormalities in community-based adults. Spine J. 2015, 15, 1593-1601. [CrossRef] [PubMed]

22. Kalichman, L.; Carmeli, E.; Been, E. The Association between Imaging Parameters of the Paraspinal Muscles, Spinal Degeneration, and Low Back Pain. Biomed. Res. Int. 2017, 2017, 2562957. [CrossRef] [PubMed]

23. Kalichman, L.; Hodges, P.; Li, L.; Guermazi, A.; Hunter, D.J. Changes in paraspinal muscles and their association with low back pain and spinal degeneration: CT study. Eur. Spine J. 2010, 19, 1136-1144. [CrossRef]

24. Freeman, M.D.; Woodham, M.A.; Woodham, A.W. The role of the lumbar multifidus in chronic low back pain: A review. PMER 2010, 2, 142-146. 